\title{
FURTHER INSIGHT INTO THE MEASURED EQUATION OF INVARIANCE
}

\author{
By Kenneth K. Mei*, Rafael Pous, Mark Prouty and Yaowu Liu \\ Department of Electrical Engineering and Computer Sciences \\ University of California, Berkeley, CA 94720
}

\section{Abstract}

The Measured Equation of Invariance (MEI) is a concept in field computation, which leads to the generation of local linear equations in lieu of absorption conditions to terminate finite difference or finite element equation. The advantage of the method is that the equations are not limited to replacing absorbing conditions, rather they can be used where there are both outgoing and incoming waves. In fact, they can be used in closed or semiclosed environment such as cavities or waveguides. And, yet unlike the integral equaiton or boundary element method, which results in global matrices, MEI gives local equations and sparse matrices. The savings in storage and computing time by MEI over conventional methods are very substantial. Since the first presentation of the method in the AP symposium in ' 92 , our results have been reproduced by other computational centers and new applications have also been achieved. The question still remains as to why the method works as well as it does, and where is the limit of the method in terms of minimizing storage and cpu time usage. And, whether there exists any limit of the target size other than the one due to the limit of the computer memory. This paper tries to answer some of those questions.

\section{Summary}

\section{THE POSTULATES}

Let the problem to be solved be a 2-D scattering problem. The Measured Equation of Invariance is based on the postulate that, the field values of an elliptical partial differential equation at a discretized node and its neighbors may be related by a general linear relation,

$$
\sum_{i=1}^{n-1} a_{i} \phi_{i}=0
$$

where $i=0$ is the node in quation and $i=1$ to $n-1$ represent the $n-1$ neighbors. Figure 1 a depicts such a node in the interior of a mesh and Fig.1b depicts a boundary node. The linear relation of (1) may be different from the finite difference or finite element equations and is,

(i) dependent on the location of the node.

(ii) geometry specific, and

(iii) invariant to the field of excitation

\section{THE METRONS}

Using the postulates as a guide, the method proceeds to find the coefficients by substituting possible solutions $\phi_{i}$ into (1). Postulate (ii) suggests that $\phi_{i}$ must be related to the target geometry, hence we use the following integrals for $k=1,2, \ldots, n-1$ 


$$
\phi^{(k)}(\bar{r})=\int G\left(\bar{r} / \bar{r}^{\prime}\right) J_{k}\left(\bar{r}^{\prime}\right) d c^{\prime}
$$

where $C$ is the surface of the target, are used to determine $a_{i}$ 's. The current density $J_{k}\left(\vec{r}^{\prime}\right)$ are named the metrons, and $\phi^{k}$ are named the measuring functions. It is noticed that the metrons are used to find the equations only, and they are not used to represent the solutions. $\phi^{k}$ of equation (2) is not the specific solution we are looking for, but, if $J_{k}$ is a smooth reasonable function, it may be the scattered field of some unknown incident field. Since postulate (iii) suggests that the $a_{i}$ 's are invariant to incident fields, there exists a wide variety of functions, which can be used as metrons. The easiest such functions are constants and sinusoidal functions of the circumferential length along the target surface, such as

$$
J_{k}=\cos \frac{2(k-1) \pi l}{L}
$$

where $L$ is the total circumferential length of the target and $l$ is the circumferential distance from a chosen point on the target. Isn't it possible that the incident field for a particular metron may not exist? It certainly is. And, it is also possible that even several $\phi^{k}$ may be linearly independent globally, they may still be linearly dependent at a few discrete points. To circumvent these difficulties, one may choose more metrons than $n-1$, and the linear equations for the $a_{i}$ 's are solved by a least square fit.

\section{THE INVARIANCE}

Among the postulates, the third postulate seems to be most incredible, and we recieved numerous requests to prove it. Actually, that postulate is not as difficult to believe as it appears. All it says is that the finite difference (or finite element)equations, which are known to be invariant to incident fields are not the only local linear relation of the fields, that lead to the solution. In other words, the coefficients $a_{i}$ 's are not unique. If they were unique, we should be able to reproduce the finite difference equaiton from $n-1$ solutions which are produced by $n-1$ different incident fields. The fact is that in all scattering problems, the finite difference equation cannot be recovered that way. As a matter of fact, the coefficients recovered that way is the Measured Equation Of Invariance.

The invariance can be most easily checked by computing experiments. It is not difficult to realize that the coefficients are numerically invariant within the accuracy of the discretization. But, one must be mindful that if high variation metrons are used, one should also use fine enough mesh to accommodate them. The conventional rule of thumb of $\lambda / 10$ may not be sufficient, because in the near field region, a rapid spacial variation of the current density begets a high spacial frequency field. A simple example is the high order modal fields of a circular cylinder.

\section{OTHER ISSUES}

A number of issues regarding the limit of the MEI approach on maximizing savings on storage and computation time will be presented. 


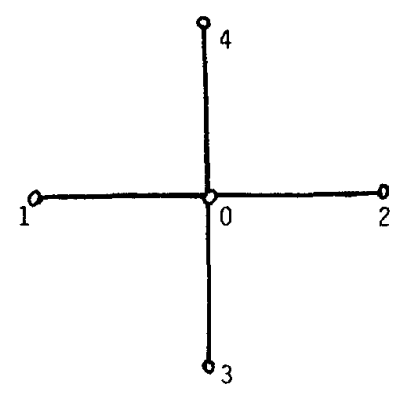

Fig. 1a An interior finite difference node and its neighbors.

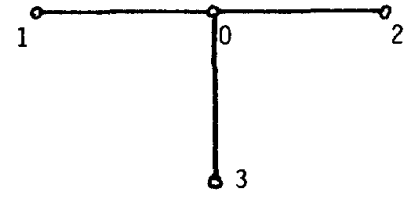

Fig. 1b A boundary finite difference node and its neighbors.

This research is supported by Califomia State MICRO and industrial sponsor Hughes Aircraft Company. 